\title{
Continuous Wave Observation of Massive Polariton Redistribution by Stimulated Scattering in Semiconductor Microcavities
}

\author{
R. M. Stevenson, ${ }^{1}$ V. N. Astratov, ${ }^{1}$ M. S. Skolnick, ${ }^{1}$ D. M. Whittaker, ${ }^{2}$ M. Emam-Ismail,,${ }^{1}$ A. I. Tartakovskii, ${ }^{3,1}$ \\ P. G. Savvidis, ${ }^{4}$ J. J. Baumberg, ${ }^{4}$ and J. S. Roberts ${ }^{5}$ \\ ${ }^{1}$ Department of Physics and Astronomy, University of Sheffield, Sheffield S3 7RH, United Kingdom \\ ${ }^{2}$ Toshiba Research Europe Ltd., Cambridge CB4 4WE, United Kingdom \\ ${ }^{3}$ Institute of Solid State Physics, RAS, 142432 Chernogolovka, Russia \\ ${ }^{4}$ Department of Physics and Astronomy, University of Southampton, Southampton SO17 1BJ, United Kingdom \\ ${ }^{5}$ Department of Electronic and Electrical Engineering, Sheffield S1 3JD, United Kingdom
}

(Received 5 July 2000)

\begin{abstract}
A massive redistribution of the polariton occupancy to two specific wave vectors, zero and $\sim 3.9 \times$ $10^{4} \mathrm{~cm}^{-1}$, is observed under conditions of continuous wave excitation of a semiconductor microcavity. The "condensation" of the polaritons to the two specific states arises from stimulated scattering at final state occupancies of order unity. The stimulation phenomena, arising due to the bosonic character of the polariton quasiparticles, occur for conditions of resonant excitation of the lower polariton branch. High energy nonresonant excitation, as in most previous work, instead leads to conventional lasing in the vertical cavity structure.
\end{abstract}

PACS numbers: 71.36.+c, 03.75.Fi, 42.50. $-\mathrm{p}$

Semiconductor microcavities permit unprecedented control of the properties of both light and matter in the solid state and of their mutual interactions [1,2,3]. This control has resulted in a new field of exciton-polariton physics, with specific properties which can be tailored by structure design. Of particular interest for the present work, the polariton coupled modes are expected to exhibit bosonlike character under excitation conditions where photoexcited electron-hole pairs retain strong excitonic character. When the bosonic character is dominant, the polariton quasiparticles are expected to exhibit scattering stimulated by final state populations in excess of unity [4]. Such behavior has been much discussed in the literature. Some early claims [5] of such "boser" behavior were either later withdrawn [5] or refuted [6]. Subsequently nonlinear behavior in $\mathrm{cw}$ luminescence experiments was attributed to final state stimulation [7]. However, the expected narrowing of the emission [8] above threshold, characteristic of macroscopic final state population, was not observed [7], and indeed it has been suggested that the results could be explained by nonlinear exciton-exciton scattering alone [9]. Finally, we have recently reported a definitive observation of stimulated scattering of polaritons in ultrafast experiments: injection of a weak probe pulse into the final state was shown to stimulate scattering from a resonantly excited pump population [10,11]. Evidence for stimulation has also been presented in a three beam experiment by Huang et al. [12].

In the present work, we show that under appropriate cw excitation conditions stimulated scattering processes are dominant in determining the energy and wave vector distribution of the polariton population. Such scattering leads to highly nonthermal distributions, peaked at particular values of wave vector. This result is surprising in view of previous work under nonresonant excitation, where although nonlinear increases of the low $k$ population with excitation were observed $[7,13,14]$, a smoothly varying distribution with $k$ was found. We show here that strong stimulation occurs for resonant excitation close to the point of inflection of the dispersion curve of the lower polariton branch. In contrast to the ultrafast case strong stimulation occurs in the presence of the excitation beam alone [11]; the process is self-stimulated with carrier-carrier interactions sufficient to permit final state populations close to unity to build up and thus to initiate stimulation. Angle resolved luminescence experiments are employed to probe the microscopic behavior. At low densities the population peaks close to the injection wave vector, with clear evidence for a relaxation bottleneck [14]. With increasing excitation the distribution shifts towards $k=0$, until above a low $k$ density of $\sim 10^{6} \mathrm{~cm}^{-2}$, corresponding to occupancies of order unity, an increase in the $k=0$ population by $\sim 2$ orders of magnitude is found. At the same time a corresponding strong increase in the population is found at a particular large wave vector, required to conserve energy and momentum in the scattering process. The observed phenomena have a number of characteristics of a Bose-Einstein condensation to the $k=0$ polariton state.

The experiments are carried out on a $3 \lambda / 2$ microcavity grown by metalorganic vapor-phase epitaxy technique (MOVPE). Three $\mathrm{In}_{0.06} \mathrm{Ga}_{0.94}$ As quantum wells are embedded at each of the two antinodes of the optical field. The vacuum Rabi splitting was $\sim 6 \mathrm{meV}$, with off resonance cavity and exciton linewidths of 0.5 and $1 \mathrm{meV}$, respectively. The measurements were carried out at a temperature of $5 \mathrm{~K}$. Excitation was provided by a Ti:sapphire laser focused to an $\sim 100 \mu \mathrm{m}$ spot. The angles $(\theta)$ of excitation and detection could be varied separately from normal incidence to $\sim 35^{\circ}$, corresponding to in-plane $k$ from 0 to $4.2 \times 10^{4} \mathrm{~cm}^{-1}(k=\omega / c \sin \theta)$. Photoluminescence 
(PL) spectra were collected by a lens (collection angle $\pm 1^{\circ}$ ) which focused the PL onto a fiber, with both lens and fiber mounted on the same rotating rail.

A series of PL spectra collected at $\theta=0$ are presented in Fig. 1(a) as a function of excitation intensity from $2 \mathrm{~W} / \mathrm{cm}^{2}$ to $2 \times 10^{3} \mathrm{~W} / \mathrm{cm}^{2}$. Results are presented for a negative detuning of $4 \mathrm{meV}$ between uncoupled cavity and exciton modes; very similar results are observed on resonance. The excitation angle is $15.5^{\circ}$, close to the point of inflection of the $E-k$ dispersion curve of the lower polariton branch, shown in Ref. [10] to be the angle at which energy and wave vector is conserved in a polaritonpolariton scattering process to low and high wave vectors [see Fig. 2(b)]. At all powers the spectra are dominated by PL from the lower polariton branch. Up to a threshold of $\sim 6 \times 10^{2} \mathrm{~W} / \mathrm{cm}^{2}$, the spectra increase linearly with power and then show a very strong superlinear increase, as shown on the light output versus excitation intensity plot of Fig. 2(a). As in the ultrafast case [10], above threshold the PL peak is shifted to higher energy by $\sim 0.5 \mathrm{meV}[15]$ and shows a marked narrowing from $0.5 \mathrm{meV}$ to a width of $0.2 \mathrm{meV}$, limited by the spectrometer resolution. As discussed below [shown in Fig. 2(b)] the shift corresponds to renormalization of the whole dispersion curve to higher

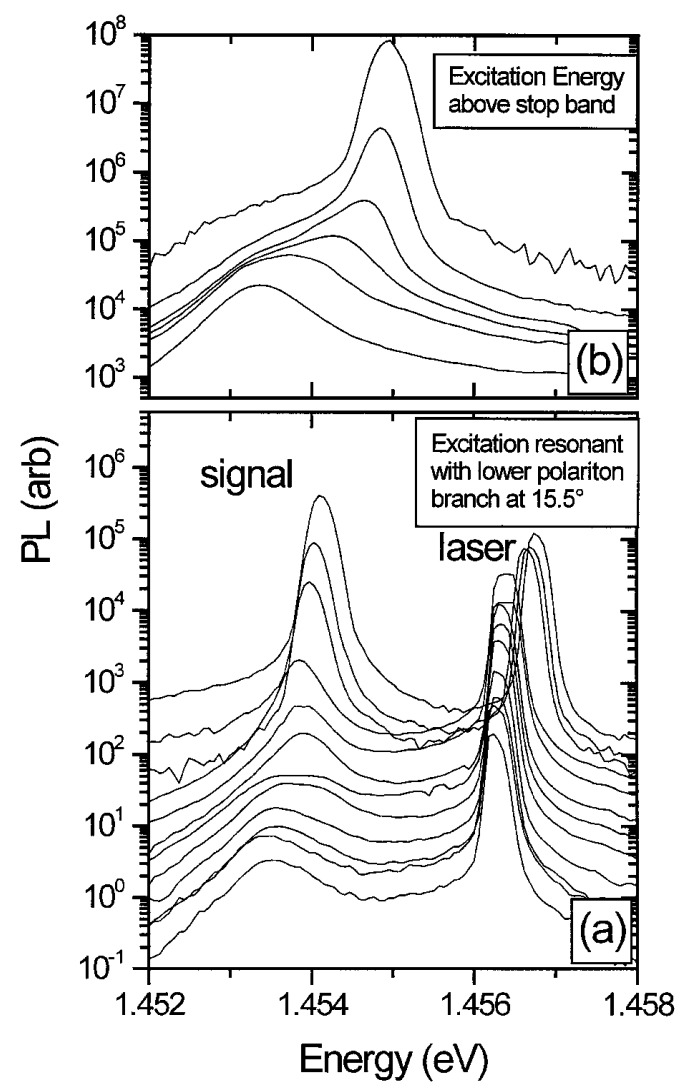

FIG. 1. (a) Photoluminescence spectra collected at $\theta=0^{\circ}$ for excitation resonant with the lower polariton branch at $15.5^{\circ}$, as a function of excitation intensity from $2 \mathrm{~W} / \mathrm{cm}^{2}$ to $2 \times$ $10^{3} \mathrm{~W} / \mathrm{cm}^{2}$. The laser is retuned to higher energy to maintain resonance above the threshold. (b) PL spectra as a function of excitation intensity for nonresonant excitation at $1.55 \mathrm{eV}$. energy. Consequently for all spectra above threshold the laser has been retuned slightly to higher energy [see Fig. 1(a)] to maximize the PL signal.

To shed light on the microscopic behavior, PL spectra were recorded as a function of detection angle, for various excitation intensities, shown in Fig. 3. Integrated intensities of the PL peaks versus $k$ are shown in Fig. 4(a). At relatively low powers $\left(<2.2 \times 10^{2} \mathrm{~W} / \mathrm{cm}^{2}\right.$, below threshold), the PL signals are maximum close to the laser energy and decrease in intensity for both positive and negative angles away from $15.5^{\circ}$, as shown in Fig. 3(a). This is an example of the polariton relaxation bottleneck observed for nonresonant excitation [14]: The escape time of polaritons from the cavity $(\sim 1 \mathrm{psec})$ is much more rapid than the relaxation to lower energy, likely to be dominated by acoustic phonon relaxation $\left(\tau_{\mathrm{ac}}>10^{-9} \mathrm{sec}\right)$ at these low powers $\left(\sim<10^{7}\right.$ polaritons $\left./ \mathrm{cm}^{2}\right)[14,16]$. With increasing power $\left[6.4 \times 10^{2} \mathrm{~W} / \mathrm{cm}^{2}\right.$, Fig. $3(\mathrm{~b})$ close to threshold], the distribution shifts to lower wave vectors, with the emergence of a small maximum at $k=0$, as seen in Figs. 3(b), 3(c), and 4(a). The overall shift to smaller $k$ is consistent with the nonlinear increase of PL

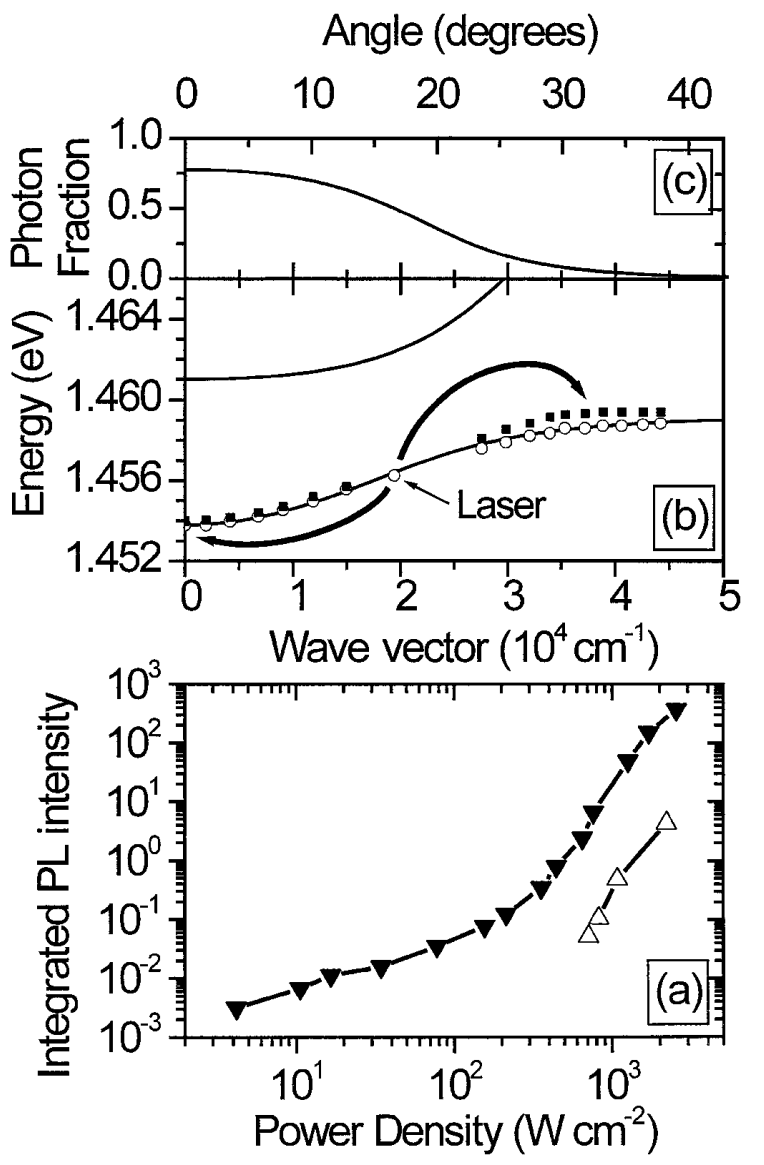

FIG. 2. (a) $k=0$ and $3.9 \times 10^{4} \mathrm{~cm}^{-1}$ PL intensities versus excitation intensity. (b) Measured lower branch dispersions below $\left(2 \times 10^{2} \mathrm{~W} / \mathrm{cm}^{2}\right.$ - open circles $)$ and above threshold $\left(2 \times 10^{3} \mathrm{~W} / \mathrm{cm}^{2}\right.$ - filled squares $)$, together with fitted upper and lower branch dispersions. (c) Photon fraction for lower branch as a function of $k$. 


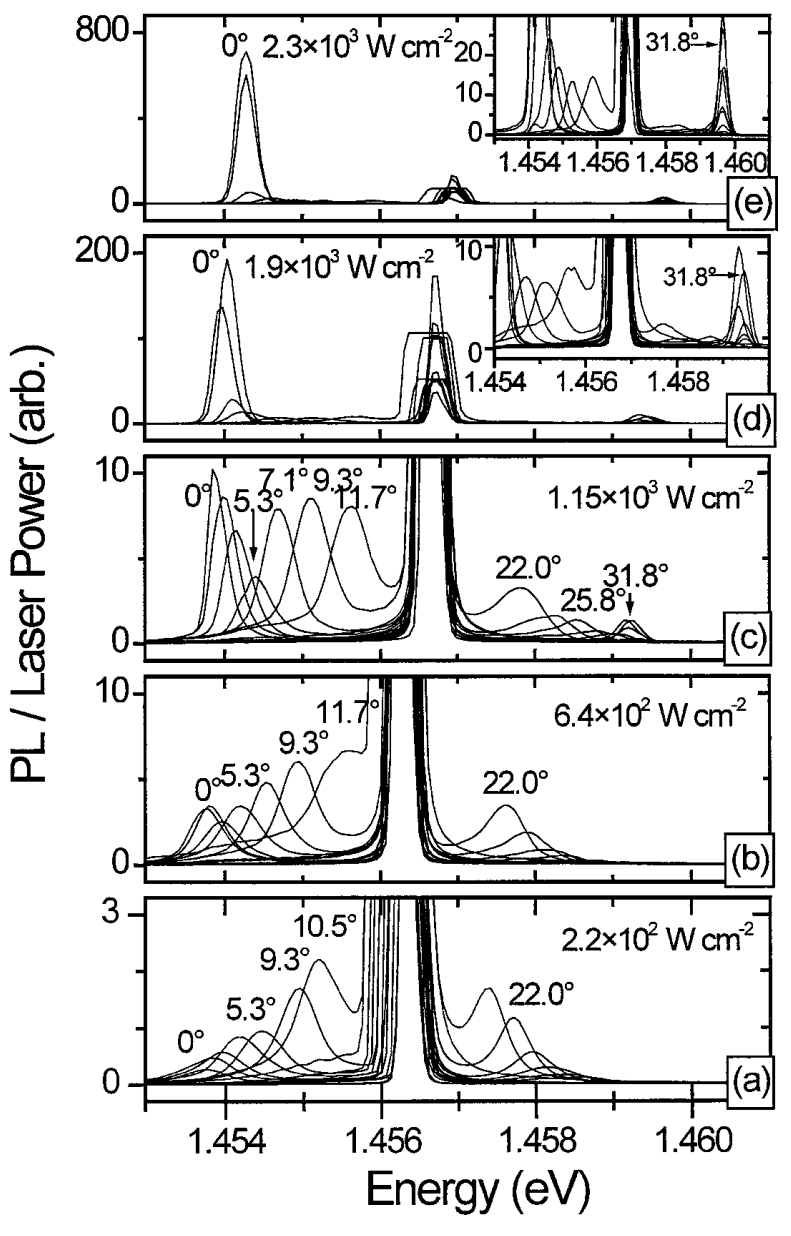

FIG. 3. PL spectra (a) below and (b) close to threshold, (c), (d), (e) above threshold as a function of detection angle. A very strong change is observed between (a) at low power where the spectra are strongest close to the excitation energy, and (d), (e) where the spectra are dominated by peaks at zero and at $32^{\circ}$. The very strong features at $\sim 1.456 \mathrm{eV}$ arise from the laser.

intensity with power at $k=0$ observed previously [7,14] under nonresonant excitation and attributed $[7,14]$ to polariton-exciton scattering [16] from the high $k$ distribution to lower wave vector. For further increase of power, a giant increase of intensity at $k=0$ and thus a massive redistribution of the polariton population to the $k=0$ state occurs. At the same time, a second strong PL signal at large $k\left(3.9 \times 10^{4} \mathrm{~cm}^{-1} \equiv 31.8^{\circ}\right)$ is also observed and exhibits the same intensity variation as the $k=0$ signal [see Fig. 2(a)]. This "idler" signal [17] conserves energy $(E)$ and $k$ in a polariton-polariton scattering process from the injected laser $E$ and $k$ to companion states at $k=0$ at $3.9 \times 10^{4} \mathrm{~cm}^{-1}$, as indicated in Fig. 2(b).

The above results enable the relative polariton densities $n_{\mathrm{pol}}$ to be deduced as a function of $k$, since $n_{\mathrm{pol}} \propto I_{\mathrm{PL}} / \tau$, where $\tau$ is the radiative lifetime of the polariton state, inversely proportional to its photon fraction, shown in Fig. 2(c). The $n_{\text {pol }}(k)$ variation thus deduced is shown in Fig. 4(b). It is notable that at high intensity the populations at $k=0$ and $3.9 \times 10^{4} \mathrm{~cm}^{-1}$ are the same within a factor of 2 , consistent with the two peaks arising from pairwise

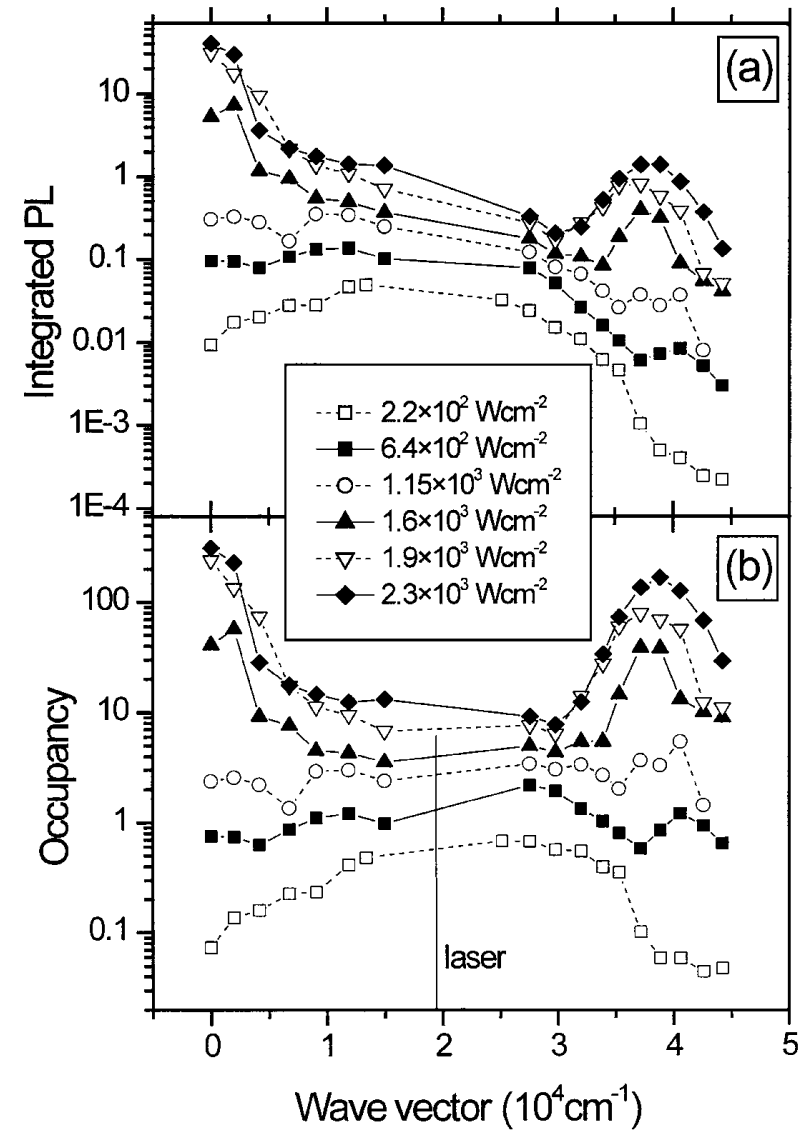

FIG. 4. (a) Integrated intensities of PL peaks as a function of in plane $k$ (angle), (b) as (a) but corrected for the photon fraction of the lower branch as a function of $k$ [from Fig. 1(c)]. By normalizing to the measured power of the $k=0$ feature above threshold, the vertical axis is labeled in terms of state occupancy.

scattering away from the resonantly created polaritons at $k \sim 2 \times 10^{4} \mathrm{~cm}^{-1}$.

We now place the $k=0$ densities of Fig. 4(b) on an absolute occupancy scale. We have measured the output power of the beam around $k=0$, a value of $1.8 \mathrm{~mW}$ being found for an input laser power density of $2.3 \times$ $10^{3} \mathrm{~W} / \mathrm{cm}^{2}$ (180 mW actual power) [18]. The $1.8 \mathrm{~mW}$ signal emitted from the $100 \mu \mathrm{m}$ spot excited corresponds to the recombination of $9 \times 10^{19}$ polaritons $/ \mathrm{cm}^{2} / \mathrm{sec}$, which given a $k=0$ lifetime of $1.7 \times 10^{-12} \mathrm{sec}$ (at $4 \mathrm{meV}$ negative detuning), corresponds to a polariton density of $1.7 \times 10^{8} \mathrm{~cm}^{-2}$. The density of states is given by $d n / d k=k / 2 \pi$, and so over the width $\Delta k$ $\left(\sim 2.6 \times 10^{3} \mathrm{~cm}^{-1}\right)$ of the near $k=0$ signal there are $(\Delta k)^{2} / 4 \pi\left(\sim 5.4 \times 10^{5}\right)$ available states $/ \mathrm{cm}^{2}$. Thus the state occupancy near $k=0$ at the highest output powers is $\sim 310$. From the relative densities of Fig. 4(b), we can then deduce the occupancy of the $k=0$ states close to threshold $\left(6.4 \times 10^{2} \mathrm{~W} / \mathrm{cm}^{2}\right)$ to be $0.8_{-0.4}^{+0.8}$. Although the errors are significant, by at least a factor of 2 , the main conclusion is clear that the threshold for stimulation of scattering to $k=0$ states (and the companion state at $3.9 \times 10^{4} \mathrm{~cm}^{-1}$ ) occurs at occupancies of order unity, consistent with bosonic final state stimulation [19]. 
It is important to demonstrate that the microcavity remains in the strong coupling limit and that a bosonic description is appropriate. First, it should be noted the polariton densities are small $\left(<10^{8} \mathrm{~cm}^{-2}\right.$ at the highest powers), significantly below the density $\left(5 \times 10^{10} \mathrm{~cm}^{-2}\right)$ where the Bose commutator of the correlated electron-hole pairs in a similar structure has been calculated to fall to a value of 0.8 [6]. Second, the $k=0$ PL peak above threshold increases in energy by only $\sim 0.5 \mathrm{meV}$, in strong contrast to the behavior for excitation above the stop band of the Bragg mirrors, where lasing occurs at $1.5 \mathrm{meV}$ above the energy of the lower polariton branch as shown in Fig. 1(b), corresponding closely to the energy of the uncoupled cavity mode, as expected in the weak coupling limit. Threshold in Fig. 1(b) occurs for a PL output signal $\sim 2$ orders of magnitude greater than in Fig. 1(a), demonstrating the clear distinction in the mechanisms and in addition the high efficiency of the bosonic stimulation process. Third, even well above threshold the polariton branch retains its characteristic dispersion, with a shift of the whole dispersion to higher energy with increasing power above threshold, shown in Fig. 2(b).

Compared to nonresonant excitation where the high $k$ population is always dominant [14], for resonant excitation of the lower branch, sufficient $k=0$ population builds up to initiate stimulation. Similarly, in our resonant ultrafast experiments, stimulation was strong only in the presence of an additional probe beam [10,11] because only a small fraction of the injection polaritons reached the $k=0$ state within the duration of the pump pulse.

We now address the question of the physical significance of the phenomena. The spectral narrowing above threshold shows that, like a laser, the system possesses a macroscopic coherence. Such a spontaneous appearance of coherence indicates that the polariton system has undergone a phase transition, consisting of the macroscopic occupation of a single mode. Since polaritons are bosons, there is some justification in describing this phase as a Bose-Einstein condensate (BEC). Support for this description is provided by clear observation of stimulated scattering of other polaritons into the macroscopically occupied mode, a characteristic property of a BEC. However, the system is far from thermal equilibrium, so although the initial buildup of the population comes from scattering down from the pump population, the phase transition is not driven by cooling. In this sense, it differs from the ideal picture of a BEC, which has a well defined temperature and a chemical potential approaching zero.

It is important to distinguish the behavior from that of a normal laser, which exhibits a macroscopic coherence, but only in the photon field. In our microcavities, the coherence is in the polariton field, which is part photon, part exciton. The existence of an exciton component is significant, as it offers the possibility for interactions to occur, possibly allowing the coherent state to be manipulated in ways not possible for a laser. Our results show a strong resemblance to a picture due to Keldysh of an idealized semiconductor laser, operating in the low density limit. He describes the appearance of a coherent polariton mode, which he calls a BEC of polaritons, due to stimulated scattering as the population builds up in the bottleneck region [20].

In conclusion, stimulated scattering of resonantly created polaritons has been reported under conditions of continuous wave excitation. The state occupancy at threshold is deduced to be of order unity, consistent with bosonic final state stimulation playing a key role in the phenomena. The strong increase of final state population corresponds to a "condensation" to a polariton mode with macroscopic occupancy, the phenomena possessing a number of similarities to Bose-Einstein condensation.

[1] C. Weisbuch, M. Nishioka, A. Ishikawa, and Y. Arakawa, Phys. Rev. Lett. 69, 3314 (1992).

[2] M.S. Skolnick, T. A. Fisher, and D. M. Whittaker, Semicond. Sci. Technol. 13, 645 (1998).

[3] G. Khitrova et al., Rev. Mod. Phys. 71, 1591 (1999).

[4] A. Imamoglu, R. J. Ram, S. Pau, and Y. Yamomoto, Phys. Rev. A 53, 4250 (1996).

[5] S. Pau et al., Phys. Rev. A 54, R1789 (1996); retracted in H. Cao et al., Phys. Rev. A 55, 4632 (1997).

[6] M. Kira, E. Jahnke, S. W. Koch, J. D. Berger, D. V. Wick, T. R. Nelson, G. Khitrova, and H. M. Gibbs, Phys. Rev. Lett. 79, 5170 (1997).

[7] P. Senellart and J. Bloch, Phys. Rev. Lett. 82, 1233 (1999).

[8] Such narrowing was observed in II-VI microcavities by Le Si Dang et al., Phys. Rev. Lett. 81, 3920 (1998). Stimulated scattering was suggested as one possible interpretation.

[9] See Ref. [3], p. 1623.

[10] P. G. Savvidis, J. J. Baumberg, R. M. Stevenson, M. S. Skolnick, D. M. Whittaker, and J. S. Roberts, Phys. Rev. Lett. 84, 1547 (2000).

[11] Weak stimulation was seen in the ultrafast case without the probe beam. It was only in the presence of the probe beam that the stimulation was strong.

[12] R. Huang, F. Tassone, and Y. Yamomoto, Phys. Rev. B 61, R7854 (2000).

[13] J. Bloch and J. Y. Marzin, Phys. Rev. B 56, 2103 (1997).

[14] A. I. Tartakovskii et al., Phys. Rev. B 62, R2283 (2000).

[15] C. Ciuti et al. (unpublished) show theoretically that the shift arises from polariton-polariton interactions.

[16] F. Tassone and Y. Yamomoto, Phys. Rev. B 59, 10830 (1999).

[17] The term "idler" is taken from the field of optical parametric oscillation (OPO); see, e.g., A. Yariv, Quantum Electronics (Wiley, New York, 1988), p. 407.

[18] We measure that $5 \%$ of the pump beam is absorbed in the sample for resonant excitation, with $20 \%$ of this light converted into the signal around $k=0$.

[19] In principle, stimulation could occur to other values of $\left(k_{\text {exc }} \pm \Delta k\right.$ ) along the dispersion curve in $E$ and $k$ conserving scattering processes. However, the $0,3.9 \times 10^{4} \mathrm{~cm}^{-1}$ values for $2 \times 10^{4} \mathrm{~cm}^{-1}$ excitation are probably favored by the larger optical feedback for $k=0$ final states.

[20] See L. V. Keldysh, in Bose-Einstein Condensation, edited by A. Griffin, D. W. Snoke, and S. Stringari (Cambridge University Press, Cambridge, England, 1995), p. 256. 